\title{
New application of non-binary Galois fields Fourier transform: digital analog of convolution theorem
}

\author{
Elizaveta S. Vitulyova1, Dinara K. Matrassulova², Ibragim E. Suleimenov ${ }^{3}$ \\ ${ }^{1,2}$ Almaty University of Power Engineering and Telecommunications named after Gumarbek Daukeyev, \\ Republic of Kazakhstan \\ ${ }^{3}$ Crimean Federal University named by V.I. Vernadsky, Simferopol, Crimea, Russian Federation
}

\begin{tabular}{l} 
Article Info \\
\hline Article history: \\
Received May 4, 2021 \\
Revised Jul 18, 2021 \\
Accepted Jul 24, 2021 \\
\hline
\end{tabular}

\section{Keywords:}

Convolution theorem

Fourier transform

Galois fields

Neural networks

\begin{abstract}
It is shown that the use of the representation of digital signals varying in the restricted amplitude range through elements of Galois fields and the Galois field Fourier transform makes it possible to obtain an analogue of the convolution theorem. It is shown that the theorem makes it possible to analyze digital linear systems in same way that is used to analyze linear systems described by functions that take real or complex values (analog signals). In particular, it is possibile to construct a digital analogue of the transfer function for any linear system that has the property of invariance with respect to the time shift. It is shown that the result obtained has a fairly wide application, in particular, it is of interest for systems in which signal processing methods are combined with the use of neural networks.
\end{abstract}

This is an open access article under the CC BY-SA license.

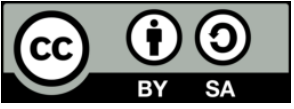

\section{Corresponding Author:}

Elizaveta S. Vitulyova

Department of Radio Engineering, Electronics and Telecommunications

Almaty University of Power Engineering and Telecommunications after Gumarbek Daukeyev

126/1 Baitursynova Street, 050013, Almaty, Kazakhstan

Email: lizavita@list.ru

\section{INTRODUCTION}

One of the main foundations of radio engineering is the apparatus of linear systems analyzing, completely built on the use of direct and inverse Fourier transforms. Research in this direction continues at the present time [1], [2] too. In particular, such fundamental concepts of radio engineering as the frequency range, as well as the transfer function of linear systems, leading to the concept of frequency response (FR), which has been and remains one of the most powerful research tools in this area [3]-[5] were created on this basis. The concept of FR is completely based on the well-defined results of the application of Fourier analysis, built on the use of harmonic functions-the convolution theorem [6]. The theorem states that under suitable conditions the Fourier transform of a convolution of two functions is the product of their Fourier transforms.

This theorem and its consequences are used not only for the analysis of time-dependent signals. In particular, the concept of the spatial frequencies spectrum in Fourier optics [7] is formulated on this basis too. Such approach gives possibility to describe the propagation of electromagnetic waves in space through the transfer function also. On the same basis, the apparatus of generalized Fourier optics [8], [9] is built, which is applicable for description of both optical and radio engineering systems (Fourier optics tools were originally developed, in particular, for solving problems in the field of radar technique [10] to describe the reflection of electromagnetic waves). We emphasize that the signals analyzed in the framework of Fourier optics are analog ones, therefore it uses Fourier transforms based on the use of harmonic functions. 
The construction of an analogue of harmonic functions intended for the description of digital signals is a non-trivial task. Their best-known analogue is the classical Walsh function, which is actively used for various applied purposes [11]-[13]. Unfortunatelly, neither classical Walsh function itself, nor its existing modifications [14]-[16] are not complete analogs of harmonic functions. Particuliarly, there are no possibility to develop, for example, the apparatus for digital linear systems analyzing in form with the same completeness and sequence in which it is developed for analog signals on the Walsh functions base.

In [17], it was proposed the following approach for processing of any digital signals that actually occur in practice: they all change in a finite range of amplitudes, and, therefore, the number of digital signal levels is also finite. Hence, it becomes possible to use Galois field, i.e. finite commutative bodies (in the algebraic sense of the last term) for digital signal processing. Let us emphasize that Galois field have been used for digital signal processing for a very long time [18]-[20], however, mainly the fields related to the binary representation of signals were used (the fields $\mathrm{GF}\left(2^{\mathrm{m}}\right)$, where $\mathrm{m}$ is an integer).

In [17] it was shown that for digital signal processing it is often advisable to use non-binary Galois field, choosing the number of their elements equal to the number of signal levels. Of course, in practice, scales corresponding to binary logic (and, accordingly, binary Galois field) are currently used, but this is nothing more than a matter of agreement. Since one can construct Galois fields GF(p), where $p$ is a prime number. This result is of interest, in particular, from the point of view of the theory of signal coding, since it is non-binary Galois field that are currently actively used in this area [21]-[23], but the area of its application is not limited to this.

In this paper, it is shown that by using functions that take values in Galois fields as a signal model, one can obtain a digital analogue of the convolution theorem. This allows us to reduce the description of any digital linear systems to finding their transfer functions in the same way as it is carried out in the classical theory of linear circuits in terms of analog signals and continuous functions.

Note that in connection with the prospect of using multivalued logics to create artificial intelligence, it is of interest not only representation of signals through non-binary Galois fields, but an analogue of the convolution theorem obtained in this work also. As emphasized in [24]-[26], intelligence, first of all, should be considered as a system of information processing, and there is no reason to assert that such systems must necessarily be built on the Aristotle's logic, which corresponds to binary one. Multi-valued logics have been actively developed throughout the twentieth century, and it is appropriate to emphasize that in the pioneering works of Lukasiewicz [27], multivalued logic was viewed precisely as an alternative to Aristotle's logic. Moreover, the functioning of neural networks, which underlie many AI systems, can be analyzed from the standpoint of coding theory [28], [29]. For this purpose, the obtained analogue of the convolution theorem is also of significant interest.

\section{ADVANTAGES OF NON-BINARY GALOIS FIELD FOURIER TRANSFORM}

Building of non-binary Galois field Fourier transform is based on the following considerations [17]. Discrete-time functions taking values in the Galois field $G F(p)$ can serve as a model for any signals that can be converted to digital form. Indeed, the scheme of dividing of the range of signal amplitudes into discrete intervals is nothing more than a matter of agreement. The standard procedure for converting a signal to digital form involves dividing the range of amplitudes into binary intervals, i.e., each discrete value of the amplitude corresponds to a certain binary number and the total number of levels is $2^{n}$. This is optional. We emphasize that digital signal processing uses namely models, i.e., real physical quantities are described by mathematical objects (continuos fuctions, for example).

To build signal models, other mathematical objects can also be used, in particular, elements of Galois fields $G F(p)$. Consequently, in order to use signal models built on Galois fields, it is sufficient to divide the range of amplitudes, which in practice is always finite, into such a number of intervals that the total number of discrete levels will be simple number.

For Galois fields, the next theorem for the sum of powers of a primitive element $\zeta$ is valid.

$$
1+\zeta+\zeta^{2}+\cdots+\zeta^{n-1}=\left\{\begin{array}{c}
n, \zeta=1 \\
0, \zeta \neq 1
\end{array}\right.
$$

Where $n=p-1$ - the number of nonzero elements in a given Galois field, which follows from the formula for a geometric progression;

$$
1+\zeta+\zeta^{2}+\cdots+\zeta^{n-1}=\frac{1-\zeta^{n}}{1-\zeta}
$$

and the fact that $\zeta^{n+1}-\zeta=0$ and $\zeta^{n}-1=0$ for any element $\zeta$ of an arbitrary Galois field containing $p=$ $n+1$ elements. 
If we consider a finite time interval, divided into discrete subintervals, then, following [17], we can define sequences of the form;

$$
w_{m}=\left(1, \theta^{m}, \theta^{2 m}, \theta^{3 m}, \ldots, \theta^{(n-1) m}\right)
$$

where $\theta$ is a primitive element, the degrees of which up to the $(n-1)$-th inclusively are give all nonzero elements of the considered Galois field, i.e. all degrees appearing in (3), de facto, do not exceed p, since the products of degrees are calculated by $\bmod (p+1)$.

With respect to the above intervals, sequences of the form (4) are direct "digital" analogs of harmonic functions (in contrast to the Walsh basis), in particular, they form a complete basis if set (3) is supplemented with a sequence consisting only of ones.

$$
w_{0}=(1,1,1,1, \ldots, 1) \text {, }
$$

This follows from formula (1), which in relation to the considered sequences (3) and (4) gives the following result;

$$
\sum_{j=0}^{j=n-1} w_{k_{1}}^{(j)} w_{k_{2}}^{(j)}=\left\{\begin{array}{l}
1, k_{1} \equiv k_{2}(\bmod (p+1)) \\
0, k_{1} \neq k_{2}(\bmod (p+1))
\end{array}\right.
$$

which allows you to go directly to the spectral representation of the signal $\vec{u}$ in the form;

$$
\vec{u}=\sum_{j=0}^{j=n-1} Z_{j} \vec{w}_{j}
$$

where elements $z_{j}$ corresponds to magnitudes of spectral components.

Multiplication of relation (7) by the vector $\vec{w}_{j}^{T}$, conjugate (in the sense of formula (6)) to $\vec{w}_{j}^{T}$, by virtue of (10), gives;

$$
\left(\vec{u}, \vec{w}_{j}^{T}\right)=\sum_{i=0}^{i=n-1} z_{i}\left(\vec{w}_{i}, \vec{w}_{j}^{T}\right)=z_{j}
$$

Relation (8) shows that obtained sequences can be interpreted as generalized Rademacher functions, and these functions constitute a complete orthogonal system by themselves, i.e., generally speaking, there is no need to pass from generalized Rademacher functions to generalized Walsh functions, especially if take into account that the prime number $p$ can be chosen large enough to process sufficiently long information packets.

We also emphasize that the amount of information required to transmit information about spectra, obtained in terms of generalized Rademacher functions, is significantly less than, for example, when using the classical Walsh basis. Indeed, the amplitude of the spectral components calculated using the Walsh basis can vary over a very wide range. Opposetely, in the case under consideration, it certainly lies in the same range as the original signal. More precisely, the spectrum is also an integer function that takes values in the same Galois field $G F(p)$ as the function that serves as a model of the original signal.

This is illustrated in Figure 1-4. Figure 1 shows the dependence of the number of deaths per week, during 16 weeks of 2020, in the state of Georgia, USA, used as an example of a model signal, in Figure 2 the same dependence, but reduced to a coarse scale corresponding to the Galois field GF(17), and in Figure 3 is its diagram corresponding to its spectrum in terms of this field.

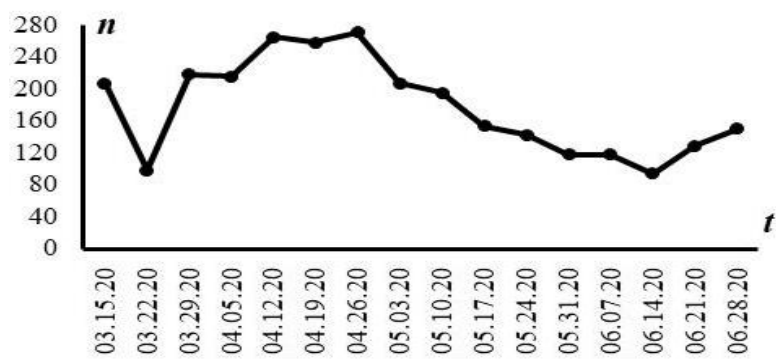

Figure 1. Model "signal" - the dependence of the number of deaths per week, during 16 weeks of 2020, in the state of Georgia, USA on time (16 values) 


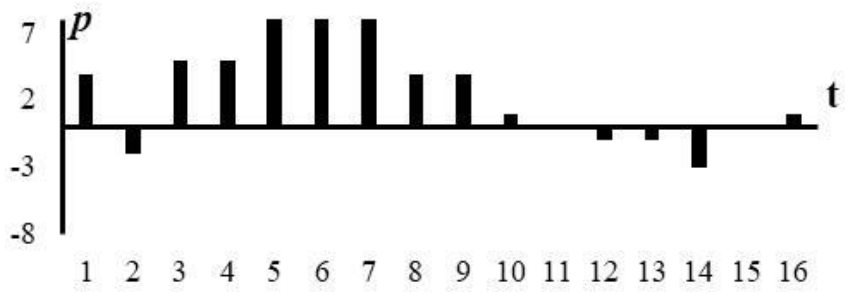

Figure 2. Model "signal" (Figure 1) in a coarse scale of 17 amplitude divisions

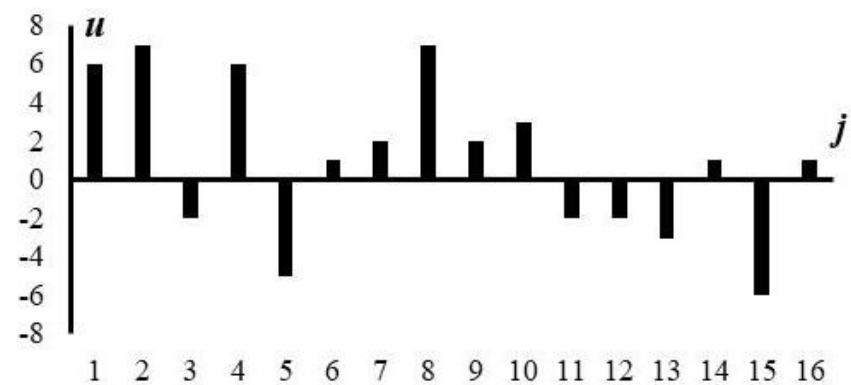

Figure 3. The spectrum of the model signal obtained using the generalized Rademacher functions constructed in the Galois field for various sequences $\mathrm{j}$, according to formula 7

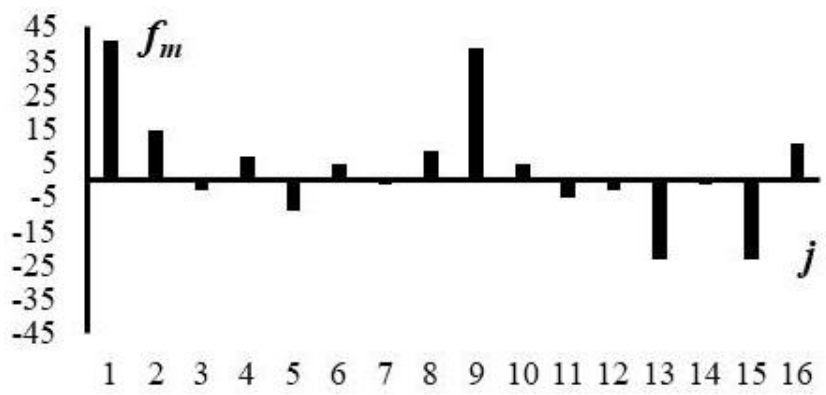

Figure 4. The spectrum of the model signal obtained using the classical walsh function

For comparison, Figure 4 shows the spectrum obtained using the classical Walsh function (the comparison is legitimate, since in both cases the sequences of 16 signal values from time are considered de facto). From a comparison of the spectra, it can be seen that the use of generalized Rademacher functions actually gives the above-mentioned advantage. Another significant advantage is the description of linear systems in the same way as in terms of harmonic functions.

\section{DESCRIPTION OF LINEAR SYSTEMS IN TERMS OF GENERALIZED RADEMACHER FUNCTIONS}

An analogue of the convolution theorem is valid in terms of generalized Rademacher functions. Let's show it. The convolution theorem states that the Fourier transform of the convolution of two functions is the product of the Fourier transforms. Let us recall the proof of this theorem in order to be able to draw the most complete analogy, as well as to clarify the significance of the developed approach for the analysis of signals taking discrete values.

The convolution of two functions $g$ and $f$, corresponding to analog signals in a complex representation, is the operation;

$$
f \otimes G=\int f\left(t_{1}\right) G\left(t-t_{1}\right) d t_{1}
$$


We emphasize that the convolution operation finds more than wide application in all areas, one way or another related to the processing and analysis of analog signals. In particular, this is due to the fact that operation (9) describes the response of any stationary linear system to a signal arriving at its input. Namely, if the system is linear and stationary, that is, its characteristics do not depend on time, then its operator $\hat{G}$ acting on a function describing an arbitrary input signal must have the property of invariance under the time shift operation;

$$
\widehat{G} f\left(t-t_{1}\right)=g\left(t-t_{1}\right)
$$

provided that,

$$
\widehat{G} f(t)=g(t)
$$

A linear operator $\widehat{G}$ acting on continuous functions and satisfying condition (10) can only have the form (9). This follows, among other things, from physical considerations. Namely, the function $G(t)$ can be interpreted, inter alia, as the response of the system to the $\delta$-shaped signal, where $\delta(t)$ is the Dirac delta function.

Indeed, in this case;

$$
\widehat{G} \delta(t)=\int \delta\left(t_{1}\right) G\left(t-t_{1}\right) d t_{1}=G(t)
$$

Consequently, relation (9) can be interpreted as a superposition of responses to a set of $\delta$-shaped signals, each of which is taken with a weight $f\left(t_{1}\right)$. In this case, as it directly follows from (9), the shift of the original signal along the time axis will lead to exactly the same shift in the response. This interpretation will also be needed later for description of linear stationary systems in terms of generalized Rademacher functions. theorem.

Applying the Fourier transform to the right-hand side of (9) gives a result known as the convolution

$$
F[G \otimes f]=\int G\left(t_{2}\right) e^{-i \omega t_{2}} d t_{2} \int f\left(t_{1}\right) e^{-i \omega t_{1}} d t_{1}=F[G] F[f]
$$

where $\omega$ is variable of frequency, $t$ - time. Symbol $F$ marks the calculation of Fourier transform.

Formula (13) is, in particular, the basis for describing any linear radio engineering circuits in terms of transfer functions. The description of signal propagation through any such circuit is reduced, as follows from (13), to the multiplication of its spectrum by the transfer function. The use of a spectrum based on generalized Rademacher functions will make it possible to determine an analogue of transfer functions in a purely discrete representation, as will be clear from what follows.

Let us consider what can be understood as an analogue of the convolution operation for such a representation. The most general analogue of formula (11) for signals in discrete representation is the record;

$$
\vec{u}=\hat{G} \vec{u}_{0}
$$

where the vectors $\vec{u}_{0}$ and $\vec{u}$, thet are given over the corresponding Galois field, correspond to the input and output signals, respectively.

An analogue of condition (10) in this case is the formula;

$$
\vec{u}(m)=\hat{G} \vec{u}_{0}(m)
$$

where $m$ marks a shift on $m$ positions.

Let us find out what the requirement for the stationarity of the system corresponds to the case when the signals are represented through Galois fields. First of all, the total number of ticks in one period must be strictly equal to the number of nonzero elements of the field $n$. Otherwise, the generalized Rademacher functions cannot be defined. Accordingly, it is advisable to consider periodic or artificially periodized signals with such a period.

An analogue of formula (12) can be represented by the following notation;

$$
\begin{gathered}
(1,0,0, \ldots, 0) \\
\downarrow \\
\left(g_{0}, g_{1}, g_{2}, \ldots, g_{n-1}\right)
\end{gathered}
$$

which implies that the response of the system to a signal of unit amplitude on the first clock cycle generates a certain signal, represented as a sequence $g_{i}$ of Galois field elements corresponding to different amplitude levels. 
Similarly, for a signal of unit amplitude acting on the second cycle, we have;

$$
\begin{gathered}
(0,1,0, \ldots, 0) \\
\downarrow \\
\left(g_{n-1}, g_{0}, g_{1}, \ldots, g_{n-2}\right)
\end{gathered}
$$

In (17) takes into account that the shift of the signal along the time axis should cause exactly the same shift in the response. Since a periodic signal with a period length $n$ is considered, this corresponds to a cyclic permutation in (17) with respect to (16).

The general formula for the response of a stationary linear system, respectively, has the form;

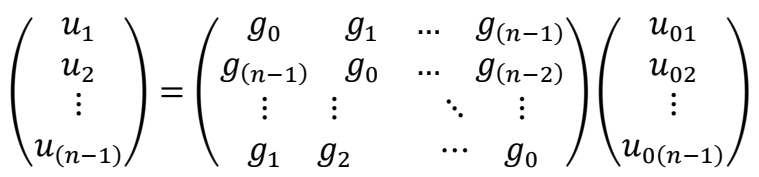

This operation can be interpreted as an analogue of the convolution operation for signals specified in the analog representation.

Let us show that generalized Rademacher functions play the same role for the analysis of linear stationary systems in discrete representation as harmonic functions play for the description of such systems in analog form.

Application of operator (18) to the sequence $\vec{w}_{1}$ gives;

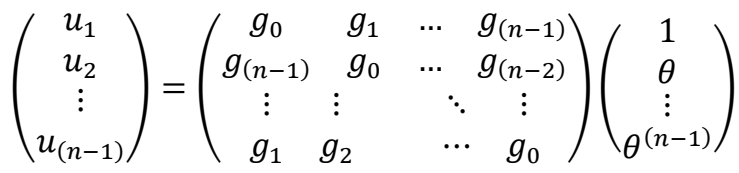

The result of multiplying each of the rows of matrix (16) by the column $\vec{w}_{1}$ is exactly described by formula (8) for calculating the spectral component of the function shifted by a certain number of clock cycles relative to the original one. Hence it follows that;

$$
\left(\begin{array}{c}
u_{1} \\
u_{2} \\
\vdots \\
u_{(n-1)}
\end{array}\right)=\left(\vec{w}_{1}, \vec{g}\right)\left(\begin{array}{c}
1 \\
\theta \\
\vdots \\
\theta^{(n-1)}
\end{array}\right)
$$

The following general formula is proved in the same way;

$$
\widehat{G}\left(\begin{array}{c}
1 \\
\theta^{k} \\
\vdots \\
\theta^{k(n-1)}
\end{array}\right)=\left(\vec{w}_{k}, \vec{g}\right)\left(\begin{array}{c}
1 \\
\theta^{k} \\
\vdots \\
\theta^{k(n-1)}
\end{array}\right)=\left(\vec{w}_{k}, \vec{g}\right) \vec{w}_{k}
$$

Let us now consider the action of the operator $\hat{G}$ on an arbitrary signal representable in terms of a basis of generalized Rademacher functions defined over a Galois field with $n+1$ elements. We represent such a signal in the form of a finite series;

$$
\vec{u}_{0}=c_{0} \vec{w}_{0}+c_{1} \vec{w}_{1}+c_{2} \vec{w}_{2}+\cdots+c_{n-1} \vec{w}_{n-1}
$$

Acting on it by the operator $\widehat{G}$ and taking into account (21), we obtain;

$$
\vec{u}=\hat{G} \vec{u}_{0}=\sum_{i=0}^{i=n-1} c_{i} \hat{G} \vec{w}_{i}=\sum_{i=0}^{i=n-1}\left(\vec{w}_{i}, \vec{g}\right) c_{i} \vec{w}_{i}
$$

Let us now apply to series (23) the operation of calculating the coefficients of a series constructed using generalized Rademacher functions. We have;

$$
\left(\vec{w}_{k}^{T}, \hat{G} \vec{u}_{0}\right)=\left(\vec{w}_{k}, \vec{g}\right) c_{k}
$$

Or, taking into account the relationship of the coefficients $c_{k}$ with the original signal, we obtain; 


$$
\left(\vec{w}_{k}^{T}, \hat{G} \vec{u}_{0}\right)=\left(\vec{w}_{k}, \vec{g}\right)\left(\vec{w}_{k}^{T}, \vec{u}_{0}\right)
$$

It can be seen that the resulting formula is a direct analogue of the formula for the Fourier transform of convolution (9), which, we emphasize once again, asserts that the Fourier transform of the convolution of two functions is the product of the Fourier transforms of these functions.

The result obtained is directly verifiable. Figure 5 shows the convolution of a signal whose values differ from zero only at the first three points, more precisely;

$$
g_{i}=\left\{\begin{array}{c}
1, i=0,1,2 \\
0, i \geq 3
\end{array}\right.
$$

with the signal shown in Figure 2.

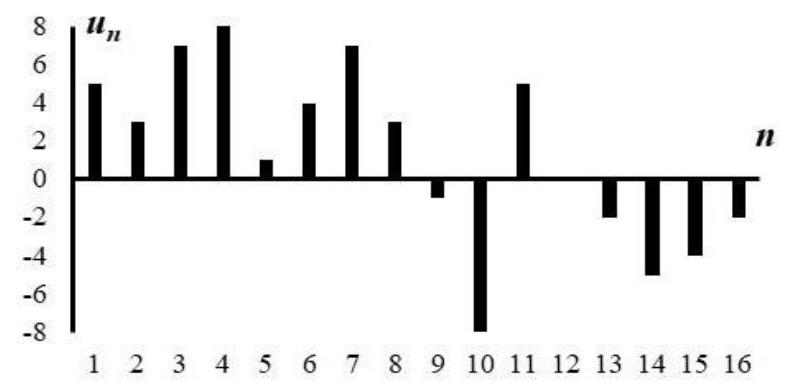

Figure 5. Convolution of the signal shown in Figure 2 with step signal (26)

In Figure 6 shows the spectrum of signal (26), and Figure 7 convolution spectrum. Direct calculation shows that the spectrum in Figure 7 is the product of the spectra in Figure 6 and 3. The resulting formula (25) makes it possible to use a direct analogue of the transfer function for digital periodic signals. Such an analogue is the set of quantities $\left(\vec{w}_{k}, \vec{g}\right)$, appearing in (25). As in classical radio engineering, the description of any linear operation that has the property of invariance with respect to a shift in time is reduced to an indication of its transfer function.

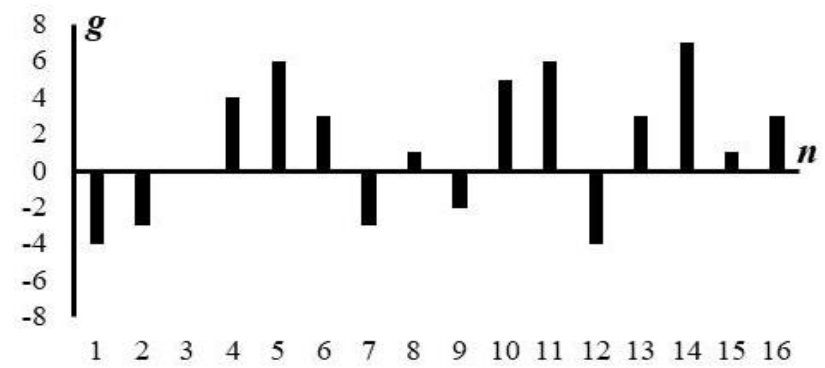

Figure 6. Signal spectrum (26) plotted in the Galois field GF (17)

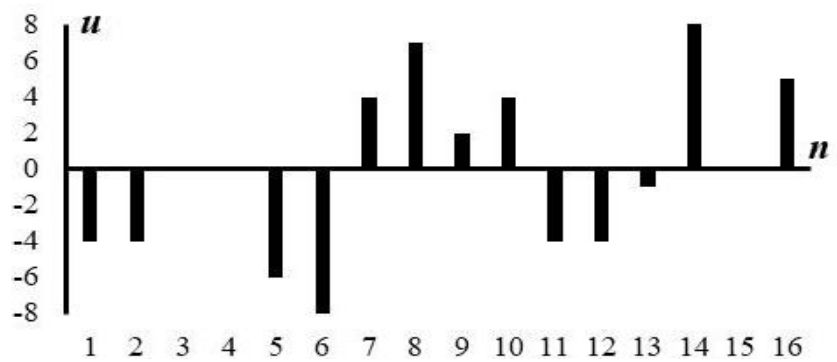

Figure 7. The spectrum of convolution of the signal (25) with the signal shown in Figure 2, obtained on the basis of formula (19), constructed in the sense of the Galois field GF (17) 


\section{CONCLUSION}

Thus, using the representation of non-binary Galois fields to represent digital signals varying in a finite range of amplitudes, it is possible to construct functions that can be interpreted as generalized Rademacher functions. They form a complete basis for any $p$-interval, which corresponds to signals given at $p$ clock cycles, where $p$ is a prime number. Such bases, in contrast to the classical Walsh basis, have many properties of harmonic functions. In particular, as shown in this paper, for the spectral representations of signals through such functions, a digital analogue of the convolution theorem is valid: the spectrum (the result of the Galois fields Fourier transform) of the convolution is the product of the spectra of the functions under its sign. It is also shown that, as in the case of analog signals, the convolution operation calculated in Galois fields describes any linear transformations over functions taking values in such fields, provided that such transformations have the property of invariance under the time shift operation.

\section{REFERENCES}

[1] Q. Kong, T. Siauw, and A. M. Bayen, "Fourier Transform," Python Programming and Numerical Methods, pp. 415-443, 2021.

[2] X. Su, R. Tao, and X. Kang, "Analysis and comparison of discrete fractional fourier transforms," Signal Processing, vol. 160, pp. 284-298, 2019, doi: 10.1016/j.sigpro.2019.01.019.

[3] V. Slyusar, "A method of investigation of the linear dynamic range of reception channels in a digital antenna array," Radio Electronics and Communications Systems of Izvestiia Vysshie Uchebnye Zavedeniia Radioelektronika, vol. 47, no. 9, pp. 20-25, 2004.

[4] F. Zhang, D. Liu, A. Liu, X. Gang, and L. Li, "Theoretical investigation on the infrasonic frequency response of measurement microphones in different venting states," Measurement, vol. 162, p. 107905, 2020, doi: 10.1016/j.measurement.2020.107905.

[5] A. Clot, J. W. R. Meggitt, R. S. Langley, A. S. Elliott, and A. T. Moorhouse, "An experimental exploration of the properties of random frequency response functions," Journal of Sound and Vibration, vol. 491, p. 115773, 2021, doi: $10.1016 /$ j.jsv.2020.115773.

[6] Deyun Wei, Qiwen Ran, Yuanmin Li, Jing Ma, and Liying Tan, "A Convolution and Product Theorem for the Linear Canonical Transform," IEEE Signal Processing Letters, vol. 16, no. 10, pp. 853-856, 2009, doi: 10.1109/LSP.2009.2026107.

[7] R. Tyson, "Fourier transforms and optics," Principles and Applications of Fourier Optics, 2014.

[8] I. Suleimenov, Y. Tolmachev, "On the possibility of generalization of Fourier optics," Optics and spectroscopy, vol. 76, pp. 893-898, 1994.

[9] I. Suleimenov, Y. Tolmachev, "Generalized Fourier optics. Reflection of monochromatic radiation from mirrors of arbitrary shape," Optics and spectroscopy, vol. 77, pp. 119-124, 1994.

[10] J. W. Goodman and M. E. Cox, "Introduction to Fourier Optics," Physics Today, vol. 22, no. 4, pp. 97-101, 1969.

[11] A Jayathilake, A. Perera, and M. Chamikara, "Discrete Walsh-Hadamard transform in signal processing," IJRIT Int. J. Res. Inf. Technol, vol.1, pp. 80-89, 2013.

[12] Y. Lu and Y. Desmedt, "Walsh transforms and cryptographic applications in bias computing," Cryptography and Communications, vol. 8, no. 3, pp. 435-453, 2015, doi: 10.1007/s12095-015-0155-4.

[13] Z. Dokur and T. Ölmez, "Heartbeat classification by using a convolutional neural network trained with Walsh functions," Neural Computing and Applications, vol. 32, no. 16, pp. 12515-12534, 2020, doi: 10.1007/s00521-020-04709-w.

[14] D. E. Dutkay and G. Picioroaga, "Generalized Walsh Bases and Applications," Acta Applicandae Mathematicae, vol. 133, no. 1, pp. 1-18, 2013, doi: 10.1007/s10440-013-9856-x.

[15] J. Irion and N. Saito, "The generalized Haar-Walsh transform," 2014 IEEE Workshop on Statistical Signal Processing (SSP), 2014, doi: 10.1109/SSP.2014.6884678.

[16] Y. Shao and N. Saito, "The extended generalized Haar-Walsh transform and applications," Wavelets and Sparsity XVIII, 2019, doi: 10.1117/12.2528923.

[17] I. Moldakhan, D. Matrassulova, D. Shaltykova and I. Suleimenov, "About some advantages of non-binary galois fields for digital signal processing, " Indonesian Journal of Electrical Engineering and Computer Science, 2021, doi: 10.11591/ijeecs.v23.i2.pp871-878.

[18] Tun Myat Aung and Ni Ni Hla, "Implementation of Finite Field Arithmetic Operations for Large Prime and Binary Fields using java BigInteger Class," International Journal of Engineering Research and Technology, vol. V6, no. 08, 2017, doi: 10.17577/IJERTV6IS080209.

[19] D. Shah and T. Shah, "Binary Galois field extensions dependent multimedia data security scheme," Microprocessors and Microsystems, vol. 77, p. 103181, 2020, doi: 10.1016/j.micpro.2020.103181.

[20] S. Sasi and L. Swarna Jyothi, "A Novel Public Key Crypto System Based on Bernstein Polynomial on Galois Fields $2 \mathrm{~m}$ to Secure Data on CFDP," Smart Intelligent Computing and Applications, pp. 639-647, 2018, doi: 10.1007/978-981-13-1927-3_67.

[21] H. Pham Thi and H. Lee, "Basic-Set Trellis Min-Max Decoder Architecture for Nonbinary LDPC Codes With High-Order Galois Fields," IEEE Transactions on Very Large Scale Integration (VLSI) Systems, vol. 26, no. 3, pp. 496-507, 2018, doi: 10.1109/TVLSI.2017.2775646.

[22] X. Liu, Y. Fan, and H. Liu, "Galois LCD codes over finite fields," Finite Fields and Their Applications, vol. 49, pp. 227-242, 2018, doi: 10.1016/j.ffa.2017.10.001. 
[23] M. Stark, G. Bauch, J. Lewandowsky, and S. Saha, "Decoding of Non-Binary LDPC Codes using the Information Bottleneck Method," ICC 2019 - 2019 IEEE International Conference on Communications (ICC), 2019, doi: 10.1109/ICC.2019.8761712.

[24] I. E. Suleimenov, O. A. Gabrielyan, A. S. Bakirov, and Y. S. Vitulyova, "Dialectical Understanding of Information in the Context of the Artificial Intelligence Problems," IOP Conference Series: Materials Science and Engineering, vol. 630, p. 012007, 2019, doi: 10.1088/1757-899X/630/1/012007.

[25] Y. S. Vitulyova, A. S. Bakirov, S. T. Baipakbayeva, and I. E. Suleimenov, "Interpretation of the category of 'complex' in terms of dialectical positivism," IOP Conference Series: Materials Science and Engineering, vol. 946, p. 012004, 2020, doi: 10.1088/1757-899X/946/1/012004.

[26] I. E. Suleimenov, Y. S. Vitulyova, A. S. Bakirov, and O. A. Gabrielyan, "Artificial Intelligence," Proceedings of the 2020 6th International Conference on Computer and Technology Applications, pp. 22-25, 2020.

[27] J. Łukasiewicz, "On Three-Valued Logic Jan Łukasiewicz. Selected Works," Ed. by L. Borkowski. Amsterdam: North-Holland, 1970.

[28] Y. S. Vitulyova, A. S. Bakirov, D. B. Shaltykova, and I. E. Suleimenov, "Prerequisites for the analysis of the neural networks functioning in terms of projective geometry," IOP Conference Series: Materials Science and Engineering, vol. 946, 2020, p. 012001, doi: 10.1088/1757-899X/946/1/012001.

[29] A. Bakirov and I. Suleimenov, "On the possibility of implementing artificial intelligence systems based on errorcorrecting code algorithms," Journal of Theoretical and Applied Information Technology, vol. 99, no. 1, pp. 83-99, 2021.

\section{BIOGRAPHIES OF AUTHORS}

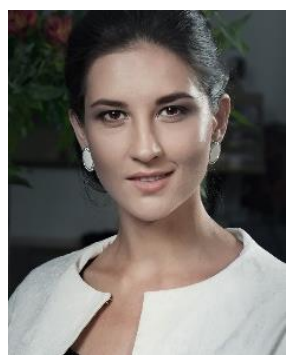

Elizaveta Vitulyova is a PhD student at the Almaty University of Power Engineering and Telecommunications after Gumarbek Daukeyev (AUPET). She received her master's degree in 2016 with a degree in radio engineering and communications at the AUPET. From 2016 to present she worked at AUPET as a senior lecturer of the department of Radio engineering, electronics and telecommunications. At the moment she is engaged in research in the field of radio engineering, electronics and telecommunications in accordance with the topic of her $\mathrm{PhD}$ thesis "Post-industrial paradigm of development of infocommunication segment in the militaryindustrial complex of the Republic of Kazakhstan".

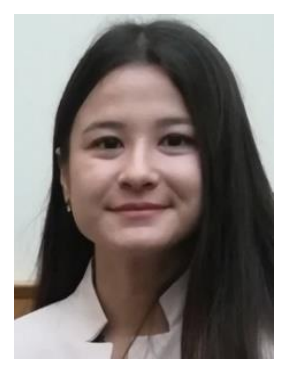

Dinara K. Matrassulova is a PhD student of the Almaty University of Power Engineering and Telecommunications. In 2015 she received a bachelor's degree and in 2017 a master's degree in "Radio engineering, electronics and telecommunications" at the Almaty University of Power Engineering and Telecommunications. She works in the telecommunications company Kcell JCS, as a senior specialist in the field of fixed internet. Actively studies telecommunications, networks, artificial intelligence, neural networks, signal processing.

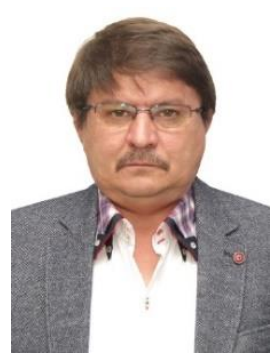

Ibragim E. Suleimenov, Professor of the Crimean Federal University named after V.I. Vernadsky (until 2020-Professor of the Almaty University of Energy and Communications). Graduated from the Physics Department of the Leningrad University named after A.A. Zhdanov in 1986; defended his thesis for the degree of candidate of physical and mathematical sciences in 1989 at the same university. In 2000, he defended his thesis for the degree of Doctor of Chemical Sciences at the Al-Farabi Kazakh National University. Academician of the National Engineering Academy of the Republic of Kazakhstan (since 2016), full professor (since 2018) according to the official certificate of the Ministry of Education and Science of the Republic of Kazakhstan. Actively develops interdisciplinary cooperation, including between natural science and humanities. He pays considerable attention to the interdisciplinary study of intelligence, both using mathematical models and at the level of philosophical interpretation. 